\title{
INFLUÊNCIA DA RAZÃO DE SOBREPOSIÇÃO NO TORQUE ESTÁTICO DA TURBINA SAVONIUS
}

\author{
L. S. BIANCHIN ${ }^{1}$, D. BECK ${ }^{2}$, D. J. SEIDEL ${ }^{3}$ \\ Instituto Federal de Educação, Ciência e Tecnologia Sul-rio-grandense \\ ORCID ID: https://orcid.org/0000-0002-5015-4302 ${ }^{1}$ \\ E-mail: lucas.sbianchin@hotmail.com \\ Submetido 10/04/2020 - Aceito 17/08/2020 \\ DOI: $10.15628 /$ holos.2020.9891
}

\section{RESUMO}

Os rotores Savonius apresentam vários parâmetros geométricos que influenciam no seu desempenho, dentre eles a razão de sobreposição, e atualmente estão sendo realizados diversos trabalhos de Dinâmica de Fluidos Computacional (CFD) para avaliar a eficiência de diferentes configurações. Dessa forma, o objetivo principal deste trabalho é analisar com o auxílio da CFD a influência de diferentes razões de sobreposição no torque estático da turbina Savonius. Para isso, foi variada a razão de sobreposição de 0 a 0,75 com passo de 0,075. As simulações de CFD foram realizadas utilizando o software Solidworks Flow Simulation 2016, o qual resolve as equações de conservação utilizando o modelo de turbulência $k-\varepsilon$ com funções de amortecimento e o método de discretização por Volumes Finitos. Através da avaliação das simulações foi possível definir que o rotor com razão de sobreposição de 0,375 é o que proporciona melhor desempenho de autopartida à turbina Savonius.

PALAVRAS-CHAVE: Turbina Eólica Savonius, Dinâmica dos Fluidos Computacional, Torque Estático, Razão de Sobreposição, Energia Eólica.

\section{INFLUENCE OF THE OVERLAP RATIO ON THE STATIC TORQUE OF THE SAVONIUS TURBINE}

\begin{abstract}
Savonius rotors present several geometric parameters that influence their performance, including the overlap ratio, and several Computational Fluid Dynamics (CFD) researchs have been performed to evaluate the efficiency of different configurations. Thus, the main objective of this research is to analyze with the help of CFD the influence of different overlap ratios on the static torque of the Savonius turbine. For this, the overlap ratio of 0 to 0.75 was varied with a pitch of 0.075 . CFD simulations
\end{abstract}

were performed using the SolidWorks Flow Simulation 2016 software, which solves the conservation equations using the $k-\varepsilon$ turbulence model with damping functions and the finite volume discretization method. Through the evaluation of the simulations it was possible to define that the rotor with an overlap ratio of 0.375 is the one that provides better self-performance to the Savonius turbine.

KEYWORDS: Savonius Wind Turbine, Computational Fluid Dynamics, Static Torque, Overlap Ratio, Wind Energy. 


\section{INTRODUÇÃO}

A energia eólica é resultante do movimento de massas de ar devido ao aquecimento irregular na crosta terrestre (Albuquerque \& Matos, 2016). O ar se aquece mais na região tropical em consequência da maior incidência dos raios de sol. Ao se aquecer, esse ar sobe, dando lugar ao ar polar, que é mais frio, e, portanto, mais denso. Além disso, o oceano se aquece de forma mais lenta que a terra, uma vez que a água tem maior capacidade de reter o calor. Dessa forma, o ar se aquece mais em determinadas regiões e menos em outras, dando origem a massas de ar com características diferentes, as quais são responsáveis por se movimentar e dar origem aos ventos (Cardoso \& Ferreira, 2015). Dentre as energias renováveis e sustentáveis, a energia eólica é a mais barata (Gawade \& Patil, 2015).

As turbinas eólicas são as responsáveis por converter a energia cinética do vento em energia mecânica. Apresentam inicialmente duas classificações quanto à orientação de seu eixo de rotação: ou são turbinas eólicas de eixo vertical (TEEVs), em que o eixo de rotação da turbina é perpendicular ao fluxo de vento; ou são turbinas eólicas de eixo horizontal (TEEHs), quando o eixo de rotação da turbina é paralelo ao escoamento (Wenehenubun, Saputra, \& Sutanto, 2015).

Um dos tipos mais conhecidos de TEEV é a Savonius, a qual tem inúmeras vantagens, como poder operar em qualquer direção do vento, ter alto torque de partida e uma geometria construtiva bastante simples, no entanto, possui baixa eficiência aerodinâmica (Jeon, Jeong, Pan, \& Ryu, 2014). Outro tipo de TEEV, chamada de Darrieus, é a que apresenta as melhores eficiências, entretanto, não é capaz de realizar a autopartida (Albuquerque \& Matos, 2016).

Um dos mais novos conceitos de TEEV é uma combinação entre as turbinas Savonius e Darrieus e tem por objetivo unir as características de ambas as turbinas para obter um rotor com bom torque de partida e bom desempenho aerodinâmico. No entanto, ainda existem poucos estudos relacionados a essa configuração de rotor e mais conhecimento é necessário para viabilizar o uso desse tipo de turbina (Chawla, Chauhan, \& Bala, 2015; Albuquerque \& Matos, 2016).

Muitos parâmetros são fundamentais no desempenho de uma turbina Savonius, principalmente em relação aos valores de coeficiente de torque. Dentre todos os possíveis, um dos mais importantes e mais estudados é a razão de sobreposição (Akwa, 2010; Kothe, 2016).

A razão de sobreposição (Rs) de uma turbina Savonius é definida como a razão entre a sobreposição (s) das pás e o comprimento da corda (c) do rotor. Aumentar a sobreposição é benéfica até certo ponto, pois proporciona recuperação de pressão na região côncava da pá de retorno, entretanto, aumentar demais esse valor ocasiona menor desempenho devido ao aumento da recirculação de ar na parte central da turbina (Kothe, 2016).

Conforme Akwa (2010), existe na literatura bastante divergência sobre qual valor de razão de sobreposição otimiza o desempenho da turbina. Os valores de sobreposição encontrados na literatura que maximizaram a potência de uma turbina Savonius variam entre 9,37\% a 22\% (Akwa, 2010; Kothe, 2016).

Segundo Roy e Saha (2013), o aumento da sobreposição é benéfico pois faz com que aumente a pressão na parte côncava da pá de retorno devido ao fluxo através da sobreposição, 
todavia, o aumento desse parâmetro geométrico também faz com que seja reduzida a pressão na parte côncava da pá de avanço. Dessa forma, em seu estudo foi obtido um valor de 0,33 para a razão de sobreposição que gerou melhor desempenho, ou seja, aquela em que ocorre o equilíbrio entre aumento da pressão na porção côncava da pá de retorno e a diminuição da pressão na parte côncava da pá de avanço. A Figura 1 ilustra o parâmetro sobreposição em uma turbina Savonius.

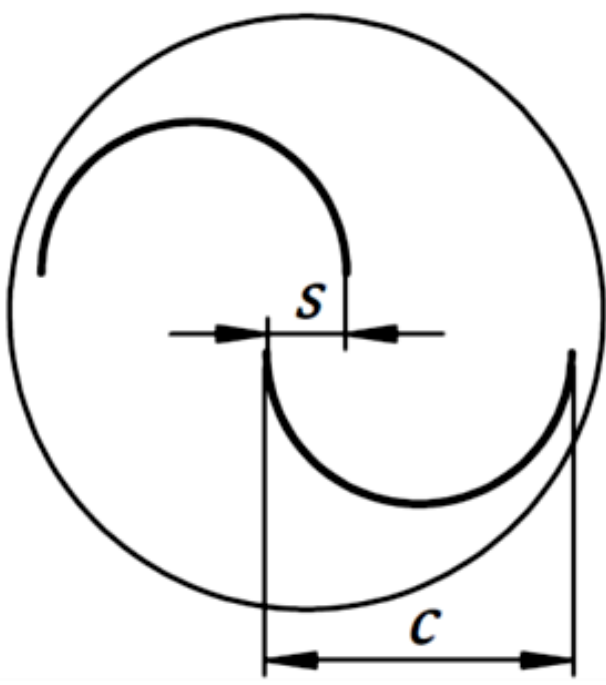

Figura 1: Sobreposição entre as pás de uma turbina Savonius.

Estudos numéricos envolvendo turbinas Savonius são um atrativo atualmente, principalmente devido à economia de material e de tempo de pesquisa. A partir das soluções de equações governantes para o campo de pressão e de velocidades, muitos dados sobre a aerodinâmica dessas turbinas podem ser obtidos e existem diversos programas computacionais atualmente que proporcionam esse tipo de estudo (Akwa, 2010).

Fundamentado nisso, este estudo busca responder: como diferentes razões de sobreposição influenciam no torque estático da turbina Savonius?

Para responder essa questão pretende-se comparar o efeito de onze diferentes razões de sobreposição no torque estático da turbina Savonius. Esses valores de torque estático serão obtidos através de simulações de Dinâmica dos Fluidos Computacional utilizando a plataforma Flow Simulation do software Solidworks 2016.

\section{METODOLOGIA}

Com o objetivo de avaliar a influência da sobreposição nos valores de torque estático, foram simuladas 11 configurações diferentes de rotores, todos com duas pás, um estágio e placas nas extremidades. Os valores de razão de sobreposição examinados foram desde a sobreposição nula (Rs=0) até o valor de Rs igual a 0,75, variando-se em 0,075 entre um valor de razão de sobreposição e outro, conforme está exibido na Figura 2. Essa gama de razões de sobreposição foi definida com o objetivo de verificar a influência da sobreposição nos valores de torque estático, uma vez que, segundo Roy e Saha (2013) e Kothe (2016), o aumento da razão de sobreposição é benéfico somente até certo ponto. 

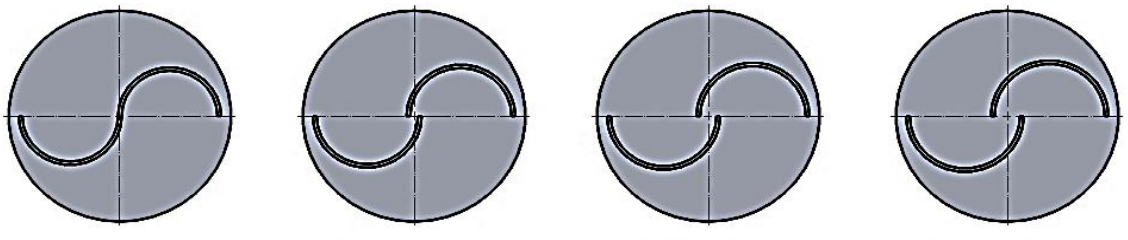

$R_{s}=0$

$R_{s}=0,075$

$$
R_{s}=0,15
$$

$R_{s}=0,225$
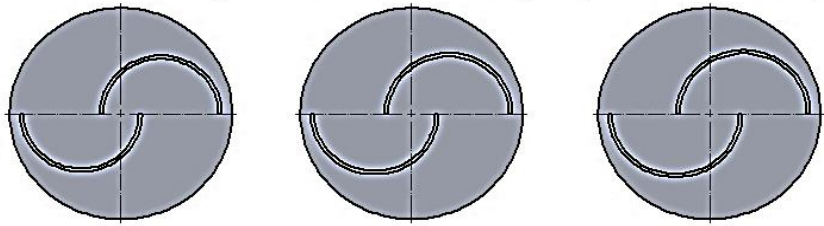

$R_{s}=0,3$

$R_{s}=0,375$

$R_{s}=0,45$

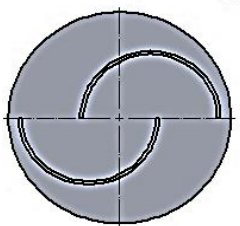

$R_{s}=0,525$

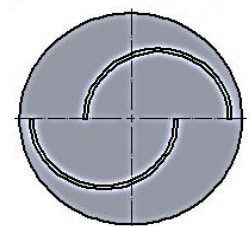

$R_{s}=0,6$

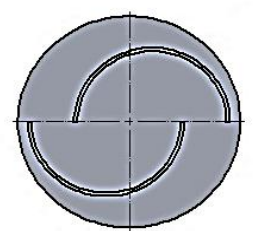

$R_{s}=0,675$

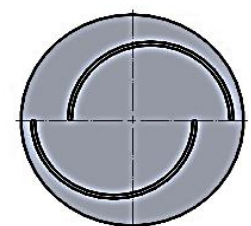

$R_{s}=0,75$

Figura 2: Diferentes tipos de turbinas Savonius analisadas em relação à razão de sobreposição.

Todas as simulações numéricas partiram de uma turbina com área de seção do rotor de $0,025 \mathrm{~m}^{2}$ e razão de aspecto de 2 , além de possuírem uma espessura de parede de $2 \mathrm{~mm}$, tanto para as pás, quanto para as placas nas extremidades. A justificativa da escolha de uma razão de aspecto de 2 vem de Akwa (2010), o qual afirma que a partir desse valor a turbina começa a apresentar maior eficiência energética. Já, a fundamentação do uso de uma área de seção do rotor de 0,025 $\mathrm{m}^{2}$ surge de dois argumentos: o primeiro, em que essa área, juntamente com a razão de aspecto de 2 , proporciona dimensões para a turbina que facilmente poderão ser reproduzidas em protótipos, com o intuito de, em trabalhos futuros, comparar os resultados obtidos neste estudo com experimentação em túnel de vento; e o segundo, o qual diz respeito ao tamanho do domínio computacional utilizado, que depende das dimensões do rotor, ou seja, quanto maior forem as dimensões do rotor, maior deverão ser as dimensões do domínio computacional, e consequentemente, maior será o tempo de processamento.

Com o propósito de melhor avaliar os valores de torque estático obtidos, cada perfil de rotor foi simulado para diferentes posições angulares em relação à direção do fluxo de vento, consoante o que é mostrado na Figura 3 , em que $\theta$ representa o ângulo entre o rotor e o escoamento e, dessa forma, foi calculado um valor de torque estático médio. Da mesma maneira que em Akwa (2010), $\theta$ foi variado de 30 em 30 graus. 

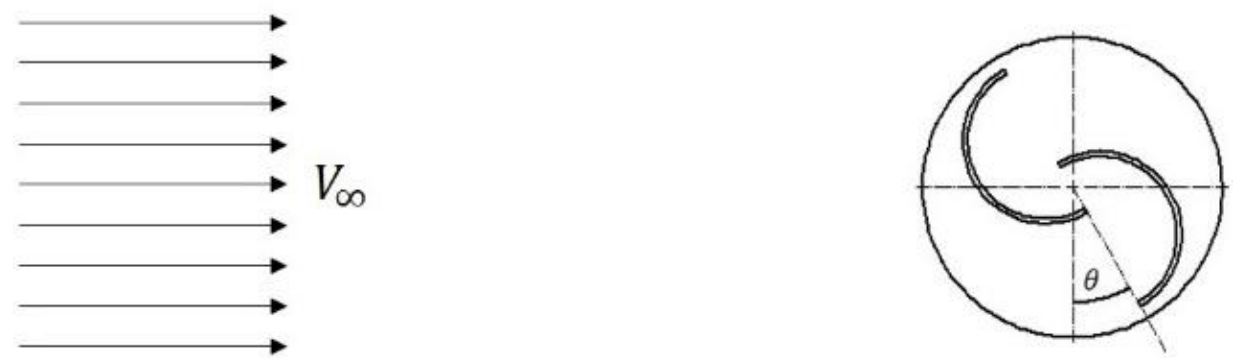

Figura 3: Posição angular do rotor em relação à direção do escoamento.

Para a realização das simulações foi utilizado o software Solidworks 2016, um programa computacional desenvolvido pela Dassault Systèmes, sendo um completo software de modelagem 3D. Ele apresenta uma plataforma denominada Flow Simulation que permite que sejam realizados cálculos de CFD utilizando o Método dos Volumes Finitos para que sejam estudados escoamentos e feitas análises do desempenho dos produtos desenvolvidos no software (Solidworks, 2016).

Com o objetivo de obter os resultados desejados, o software Solidworks resolve simultaneamente as equações da continuidade, da conservação do movimento, equações de estado e as duas equações referentes ao modelo de turbulência utilizado pelo programa, denominado de $k-\varepsilon$ com funções de amortecimento, o que permite que sejam analisados fluxos laminares, transitórios ou turbulentos (Solidworks, 2016).

Conforme Çengel e Cimbala (2012), o modelo de turbulência $k-\varepsilon$ requer que sejam especificados os valores da energia cinética turbulenta e da taxa de dissipação turbulenta, entretanto, muitas vezes é mais apropriado especificar a intensidade da turbulência (IT) e a escala de comprimento turbulento (I). A intensidade da turbulência representa a razão entre as velocidades turbulentas e as velocidades médias enquanto que a escala de comprimento turbulento significa a escala de comprimento dos vórtices gerados pela turbulência.

De acordo com Çengel e Cimbala (2012), na falta de informações sobre o comportamento do escoamento, pode-se usar valores de IT como $10 \%$ e de I como metade do comprimento característico do objeto que experimenta o fluxo. Dessa forma, para a realização das simulações o valor da escala de comprimento turbulento utilizada foi de metade do diâmetro da turbina, ou seja, $55,9 \mathrm{~mm}$.

Outros dados de entrada são necessários para a realização das simulações, como os de velocidade não perturbada, temperatura e pressão do ar. Os valores utilizados foram de $7 \mathrm{~m} / \mathrm{s}$ para a velocidade, 101325 Pa para a pressão e $293,15 \mathrm{~K}$ para a temperatura. A velocidade e a pressão servirão como condição de contorno para a execução dos cálculos pelo software. O Solidworks (2016) chama esses valores de condições ambientes, uma vez que as simulações serão de escoamento externo.

O valor de $7 \mathrm{~m} / \mathrm{s}$ para a velocidade foi utilizado pois está compreendido entre as velocidades utilizadas para avaliar o torque estático em outros trabalhos. Roy e Saha (2013) avaliaram o torque estático para as velocidades de $5,57 \mathrm{~m} / \mathrm{s}, 6,69 \mathrm{~m} / \mathrm{s}, 8,35 \mathrm{~m} / \mathrm{s}$ e $10,44 \mathrm{~m} / \mathrm{s}$, Oliveira (2014) utilizou uma velocidade de $6 \mathrm{~m} / \mathrm{s}$ e Kothe (2016) utilizou 7,6 m/s. Além disso, Hayashi, Li e Hara (2005) verificaram que a velocidade do escoamento tem pouca influência nos valores do coeficiente de torque estático. Ainda, é utilizada a pressão atmosférica de 101325 Pa e a temperatura de $293,15 \mathrm{~K}$, visto que esses são os valores considerados padrões ao nível do mar 
(Shepherd \& Zhang, 2017).

As dimensões utilizadas para o domínio computacional são baseadas no trabalho de Akwa (2010), que as atribui valores múltiplos das dimensões do rotor. A Figura 4 exibe as dimensões variadas para a análise do tamanho do domínio computacional. Os valores Z+, Z- e X são múltiplos do diâmetro (D) do rotor enquanto $Y$ é múltiplo da altura $(H)$. Ainda, o escoamento de ar percorre o domínio no sentido negativo do eixo $Z$.

A análise do tamanho do domínio computacional consistiu de duas etapas: a primeira, na qual foi avaliada a altura $Y$, mantendo-se constante as demais dimensões; e a segunda, na qual foram examinados os valores de $X, Z+e$ Z-, utilizando a altura $Y$ que melhor se adequou ao estudo, conforme a primeira etapa. Foi chegado à conclusão de que o domínio com as dimensões de $X, Z+$ e Z- iguais a, respectivamente, 18,9 e 27 diâmetros e de $\mathrm{Y}$ igual a $5 \mathrm{H}$ será o utilizado para as simulações deste estudo.

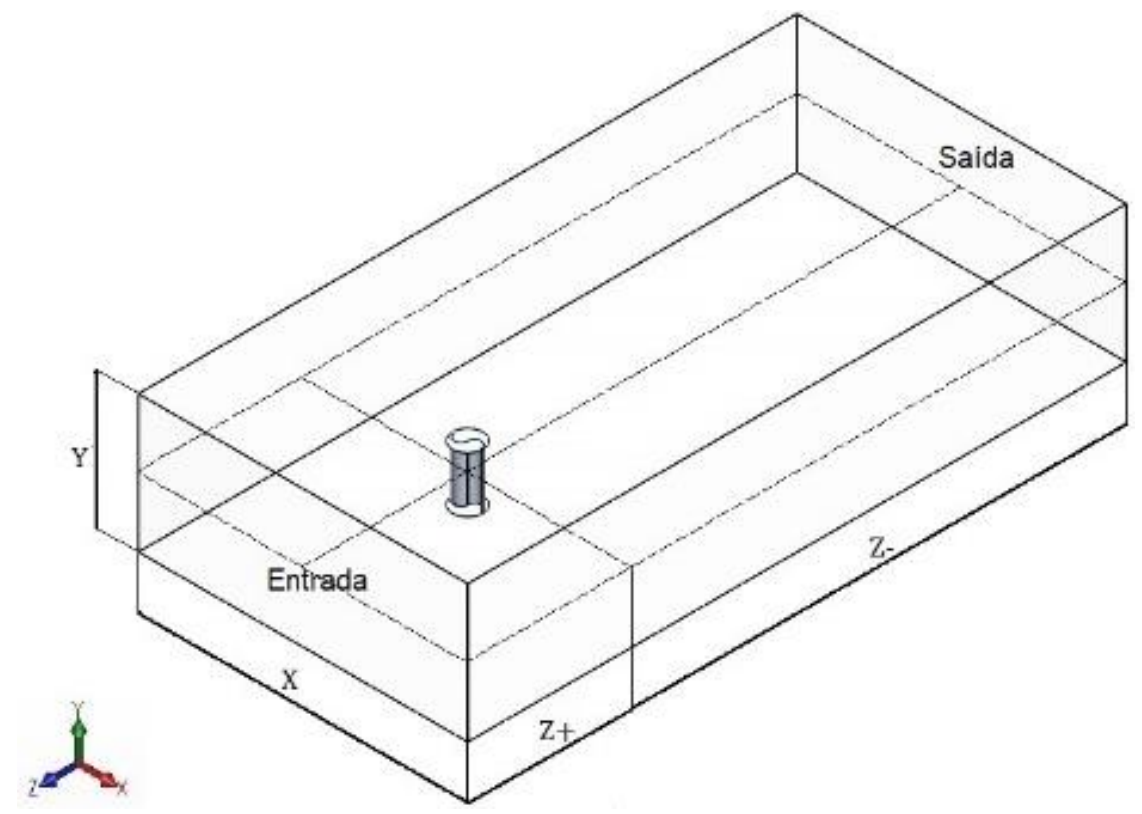

Figura 4: Dimensões a serem avaliadas para determinação do tamanho do domínio computacional.

A partir dessas considerações, avaliou-se a influência das placas nas extremidades no torque estático da turbina Savonius.

\section{RESULTADOS E DISCUSSÕES}

Com o objetivo de comparar os valores do coeficiente de torque estático médio para as diferentes razões de sobreposição foi desenvolvida a Figura 5. 


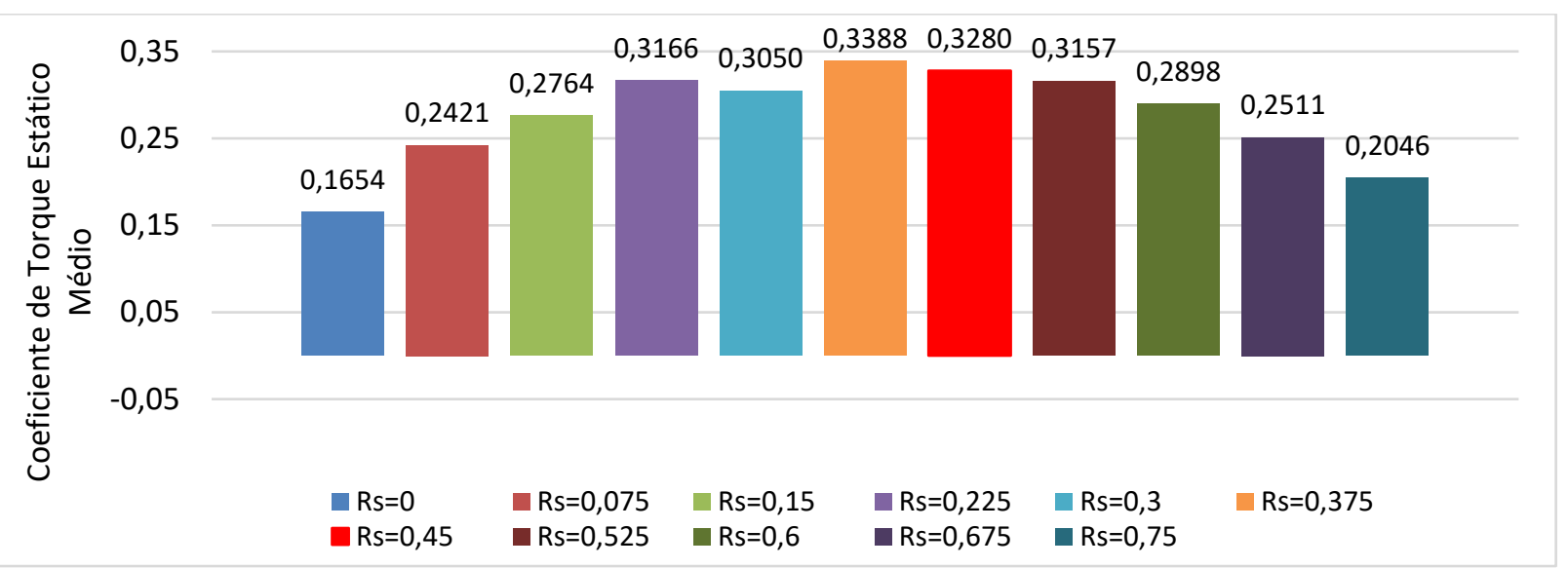

Figura 5: Variação do coeficiente de torque estático médio de acordo com a razão de sobreposição das pás da turbina Savonius.

Pela Figura 5 é possível observar que o coeficiente de torque estático pode até dobrar de valor com uma sobreposição de 0,375 em relação à condição sem sobreposição. Resultado semelhante foi obtido por Roy e Saha (2013), em que os autores obtiveram um máximo valor de torque estático para uma razão de sobreposição de 0,33. No entanto, embora o presente estudo e o de Roy e Saha (2013) tenham chegado a resultados semelhantes para o coeficiente de torque estático, outros estudos, que avaliaram a influência da razão de sobreposição na eficiência de potência da turbina Savonius, divergem entre si. Por exemplo, Fujisawa, 1992 e Akwa, Silva Júnior e Petry (2012) concluíram que a melhor razão de sobreposição é de 0,15 , enquanto que Alexander e Holownia (1978) e Sharma, Gupta e Biswas (2014) determinaram os valores ótimos como sendo 0,0937 e 0,22, respectivamente. Dessa forma, além dos resultados desses estudos terem divergido entre si, divergem também dos valores obtidos pelo presente trabalho e pelo de Roy e Saha (2013). No entanto, os outros trabalhos foram mais voltados à eficiência de geração de energia, enquanto que esses analisam a eficiência de autopartida. Dessa maneira, devido à divergência nos resultados, novos estudos podem ser realizados com o objetivo de comparar a influência da sobreposição entre as pás tanto para a eficiência de autopartida como para a eficiência de geração de energia.

Conforme a Figura 5, o aumento da razão de sobreposição até determinado ponto proporciona o aumento do torque estático. Isso ocorre, pois, a passagem de ar para a região côncava da pá de retorno é facilitada (Kothe, 2016). Assim, nessa porção do escoamento, conforme se aumenta a sobreposição, ocorrem maiores valores de pressão do que na condição de sobreposição nula. Essas maiores pressões contrabalançam a força gerada na parte convexa da pá de retorno - que é contra ao movimento - gerando maior eficiência.

As Figuras 6 e 7 apresentam, respectivamente para as sobreposições de 0 e de 0,375, as distribuições de pressão no centro do rotor com a turbina na posição angular de $0^{\circ}$. A principal diferença entre as distribuições de pressão nas duas imagens está na porção côncava da pá de retorno, uma vez que o aumento da razão de sobreposição faz com que haja maior pressão nessa região da turbina. 


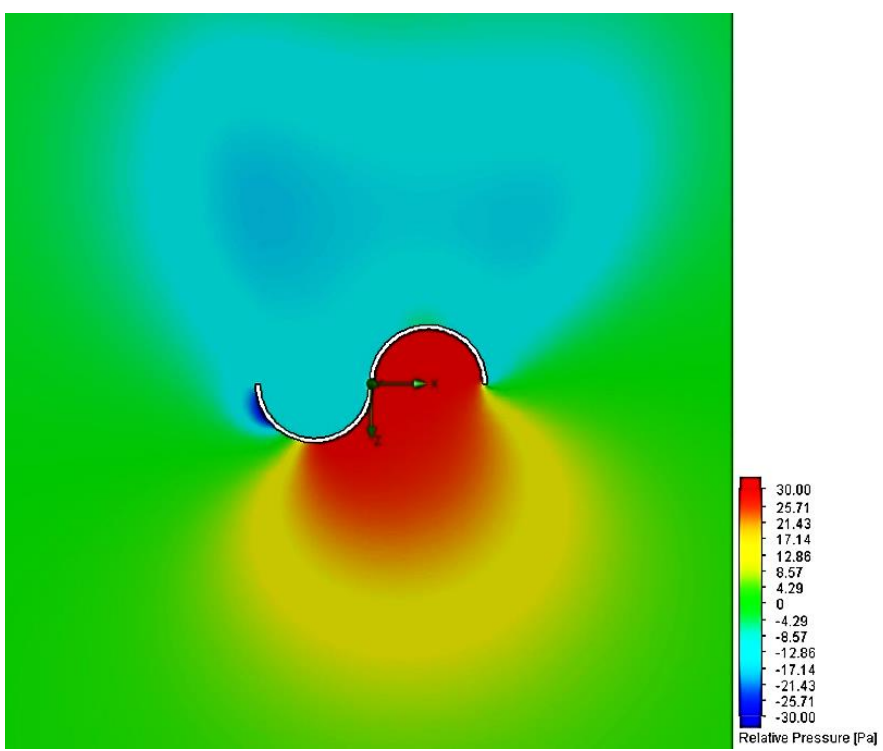

Figura 6: Distribuição de pressão para uma turbina Savonius com razão de sobreposição zero e vento incidindo a $0^{\circ}$.

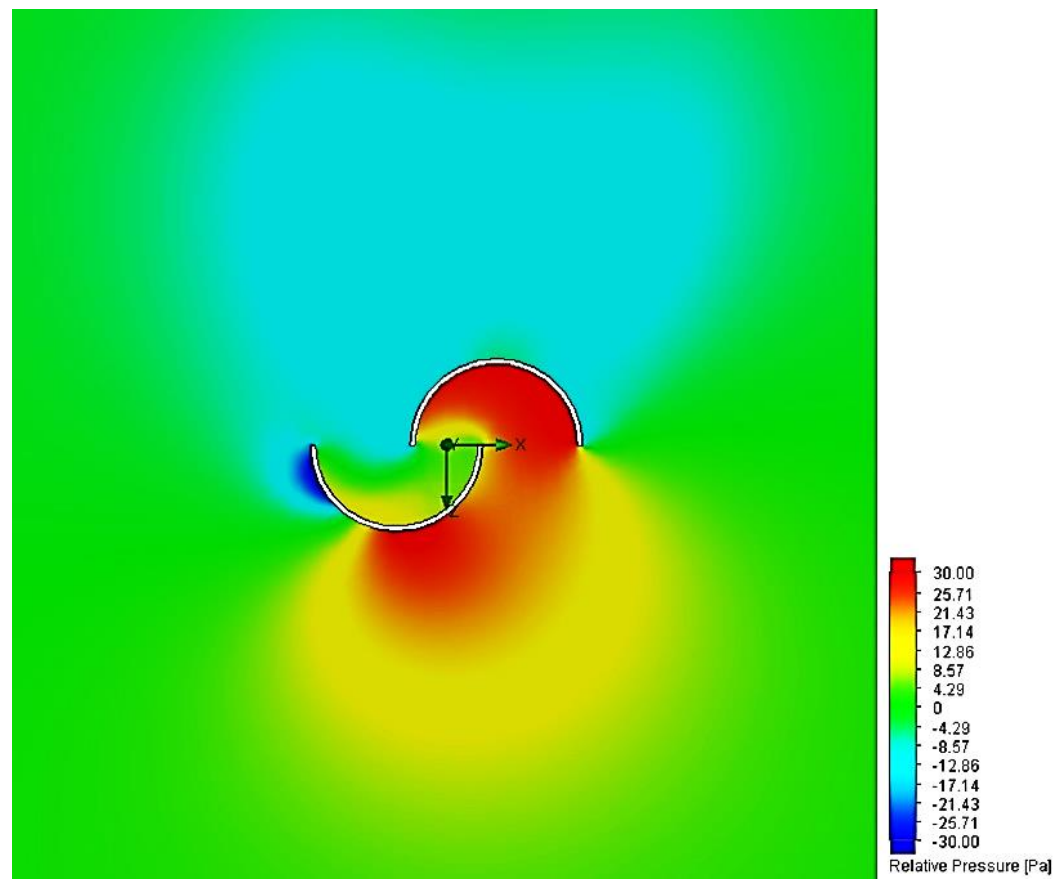

Figura 7: Distribuição de pressão para uma turbina Savonius com razão de sobreposição 0,375 e vento incidindo a $0^{\circ}$.

Esse aumento de pressão, e consequentemente, aumento no desempenho da turbina ocorre, pois, a presença da sobreposição entre as pás permite que ocorra um fluxo do escoamento da região côncava da pá de avanço para a região côncava da pá de retorno (Roy \& Saha, 2013). Isso pode ser confirmado pela comparação entre as Figuras 8 e 9. 


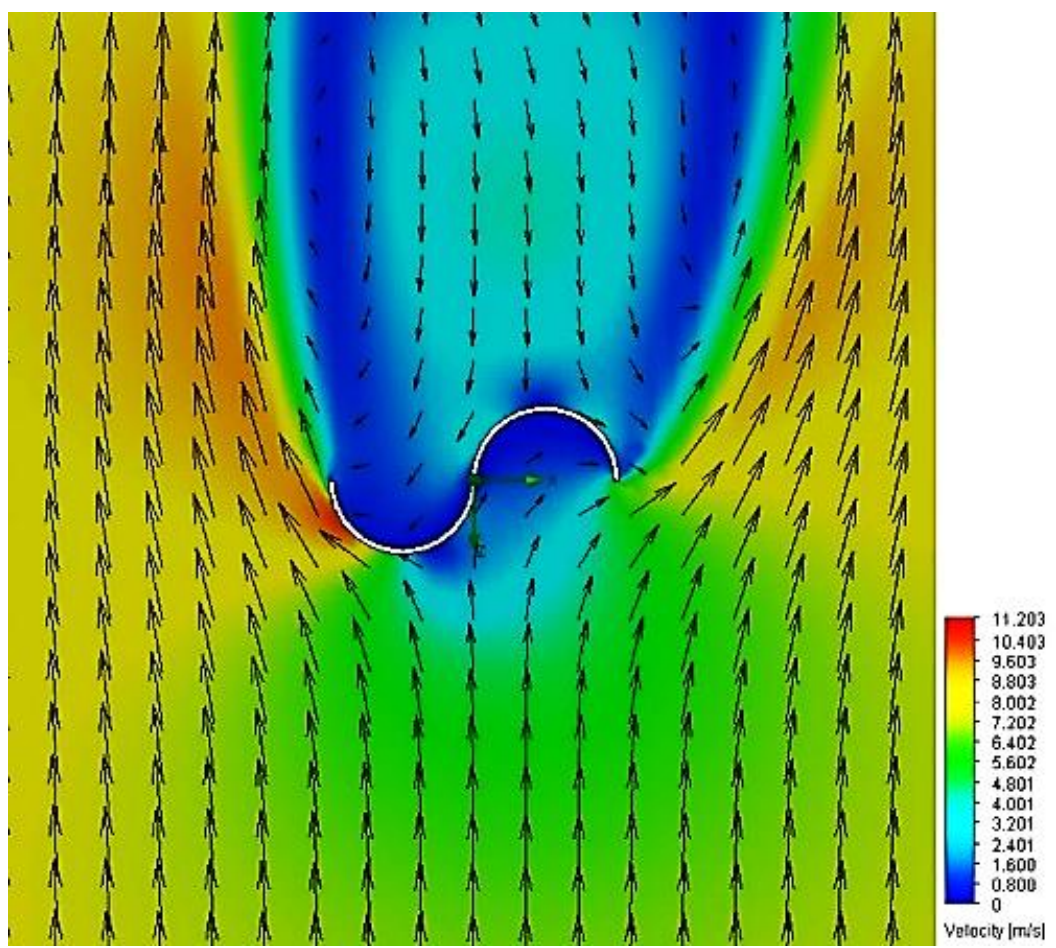

Figura 8: Campo vetorial de velocidades para uma turbina Savonius com razão de sobreposição zero e vento incidindo a $0^{\circ}$.

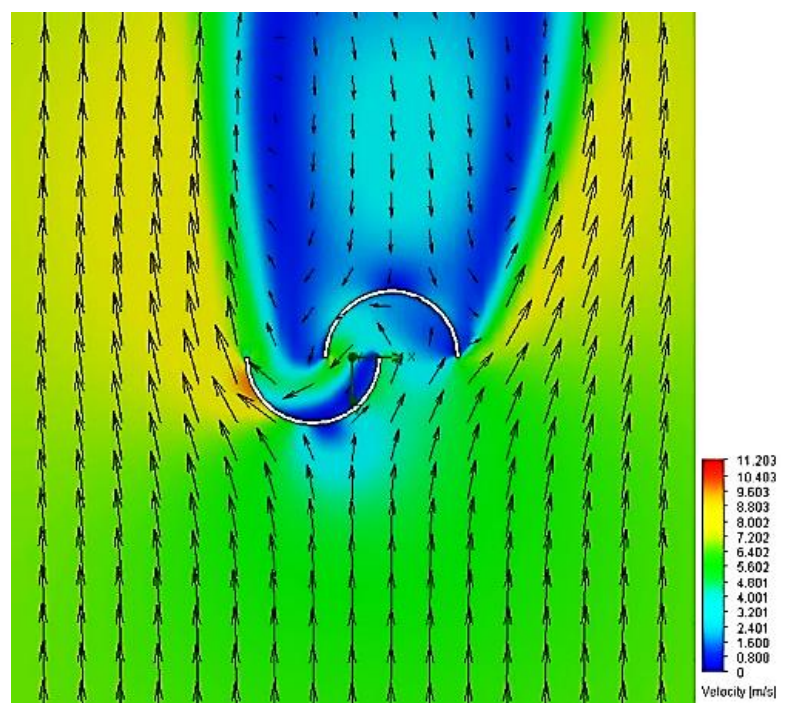

Figura 9: Campo vetorial de velocidades para uma turbina Savonius com razão de sobreposição 0,375 e vento incidindo a $0^{\circ}$.

As Figuras 8 e 9 apresentam, respectivamente para as sobreposições de 0 e de 0,375, as distribuições e o campo vetorial de velocidade no centro da turbina na posição angular de $0^{\circ}$. É possível verificar que na Imagem 8 o escoamento passa pela região côncava da pá de avanço e, devido à sobreposição ser diferente de zero, é direcionado para a porção côncava da pá de retorno (Akwa, 2010), fazendo com que, aumente a pressão na região côncava da pá de retorno (comparação entre as Figuras 8 e 9), diminua a força de arrasto nessa lâmina da turbina e consequentemente proporcione maior eficiência. 
Entretanto, aumentar a sobreposição é benéfico somente até certo ponto (Roy \& Saha, 2013). Conforme pode-se notar pela Figura 10, que ilustra o campo de pressão para a razão de sobreposição de 0,75 , grandes sobreposições fazem com que seja dificultada a incidência do vento na parte côncava da pá de avanço, diminuindo a pressão nessa região dessa lâmina e proporcionando menores valores de torque estático.

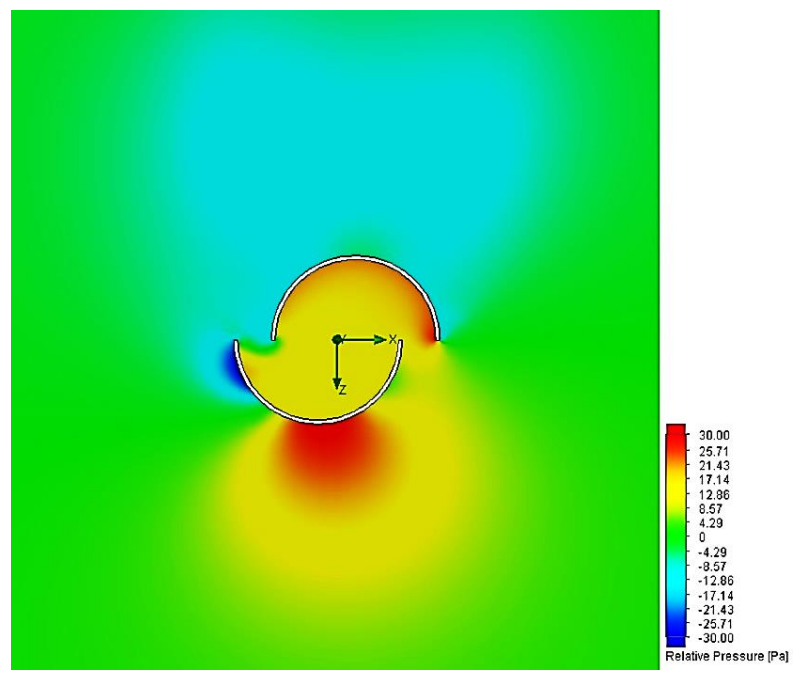

Figura 10: Distribuição de pressão para uma turbina Savonius com razão de sobreposição 0,75 e vento incidindo a $0^{\circ}$.

Dessa forma, pode-se dizer que o aumento da razão de sobreposição até certo ponto proporciona melhor eficiência de autopartida para o rotor Savonius devido à passagem de ar para a porção côncava da pá de retorno. Nesse estudo, a razão de sobreposição que gerou melhor desempenho foi a de 0,375.

Com o objetivo de exemplificar a relação da pressão nas pás da turbina Savonius com as diferentes razões de sobreposição foi desenvolvida a Figura 11. Esse gráfico mostra que os primeiros aumentos de sobreposição proporcionam aumentos consideráveis da pressão na parte côncava da pá de retorno, influenciando no ganho de desempenho. No entanto, essa pressão tende a se estabilizar para maiores valores de sobreposição. 


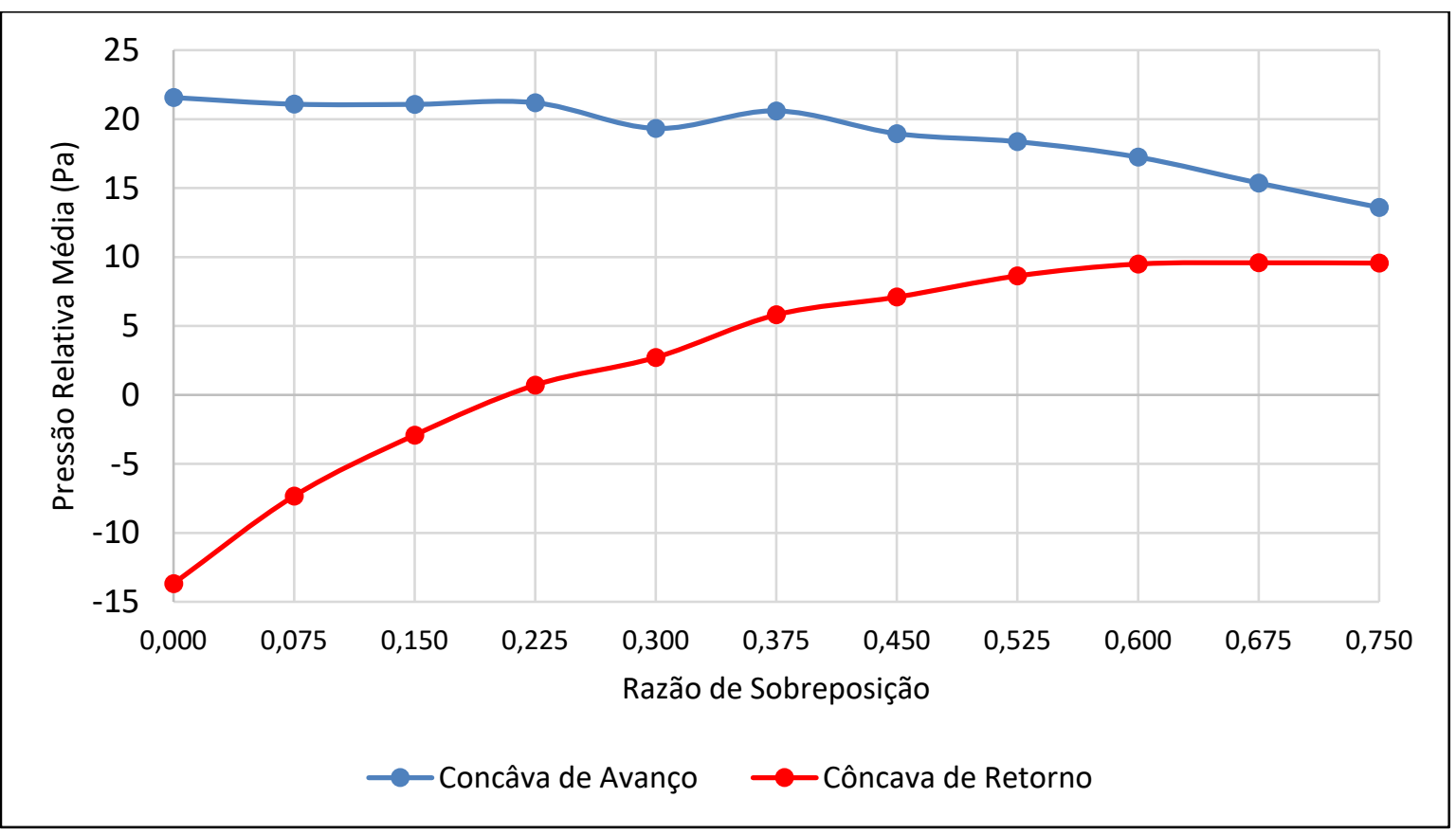

Figura 11: Variação da pressão relativa média em função da razão de sobreposição para as regiões côncavas das pás de avanço e de retorno.

Em relação à pressão na parte côncava da pá de avanço, observa-se pela Figura 11 que ela praticamente se mantém constante até a razão de sobreposição de 0,375 (com exceção da razão de sobreposição de 0,3 ), que foi aquela que proporcionou maior eficiência, e começa a diminuir gradativamente a partir dessa sobreposição, sendo um dos fatores responsáveis pela diminuição da eficiência a partir da razão de sobreposição de 0,375.

Dessa forma, a diminuição da eficiência em autopartida da turbina Savonius a partir da razão de sobreposição de 0,375 tem influência de dois fatores: um deles, que é a tendência em estabilizar a pressão na parte côncava da pá de retorno; e o outro, que é a diminuição da pressão na porção côncava da pá de avanço. Vale ressaltar que a Figura 11 é baseada na pressão média sobre as pás da turbina eólica, no entanto, o torque estático depende das forças aplicadas nas pás da turbina e também das posições dessas forças em relação ao centro do rotor. Dessa forma, embora a Figura 11 forneça uma visão inicial e permita uma discussão quanto à relação entre pressão nas placas e razão de sobreposição, essa análise é mais complexa, uma vez que a real pressão nas pás da turbina eólica varia com a posição ao longo da superfície da lâmina.

\section{CONCLUSÃO}

Neste trabalho foram desenvolvidas simulações de Dinâmica de Fluidos Computacional (CFD) utilizando a versão educacional do software Solidworks Flow Simulation 2016 com o principal objetivo de analisar a influência de diferentes razões de sobreposição nos valores de torque estático, e consequentemente, no desempenho de autopartida da turbina Savonius.

Em relação aos efeitos da razão de sobreposição, foi observado que o aumento desse parâmetro até o valor de 0,375 proporciona o melhor valor de coeficiente de torque estático médio 
$(0,3388)$, sendo duas vezes superior à condição sem sobreposição $(0,1654)$. Esse aumento na eficiência de autopartida ocorre, pois, a presença da sobreposição entre as pás faz com que ocorra um fluxo do escoamento da porção côncava da pá de avanço para a região côncava da pá de retorno, aumentando a pressão na porção côncava, e consequentemente, diminuindo os efeitos do arrasto na pá de retorno. No entanto, o aumento da razão de sobreposição a partir do valor de 0,375 faz com que ocorra perda de desempenho pois o ar começa a ter dificuldade para incidir na parte côncava da pá de avanço. Para a maior razão de sobreposição avaliada, que foi a de 0,75 , foi obtido um coeficiente de torque estático médio de 0,2046 , bastante inferior àquele observado para a razão de sobreposição de 0,375.

\section{REFERÊNCIAS}

Akwa. (2010). Análise aerodinâmica de turbinas eólicas Savonius empregando Dinâmica dos Fluidos Computacional. Tese de Doutorado, Universidade Federal do Rio Grande do Sul, Porto Alegre, RS, Brasil.

Akwa, J. V., Júnior, G. A., \& Petry, A. P. (2012). Discussion on the verification of the overlap ratio influence on performance coefficients of a Savonius wind rotor using computational fluid dynamics. Renewable Energy, 38, pp. 141-149.

Albuquerque, I. M., \& Matos, F. F. (2016). A Characterization of Vertical Axis Wind Turbines. IEEE Latin America Transactions, 14(10), 4255-4260.

Alexander, A., \& Holownia, B. P. (1978). Wind tunnel tests on a Savonius rotor. Journal of Industrial Aerodynamics, 343-351.

Cardoso, J. M., \& Ferreira, D. H. (2015). Equações matemáticas e conceitos físicos aplicados a uma turbina eólica vertical de pequeno porte. Revista Brasileira de Iniciação Científica, 2(3), 42-59.

Çengel, Y. A., \& Cimbala, J. M. (2012). Mecânica dos Fluidos: fundamentos e aplicações. Porto Alegre: AMGH.

Chawla, S., Chauhan, A., \& Bala, S. (2015). Parametric Study of Hybrid Savonius-Darrieus Turbine. Proceedings of 2015 RAECS.

Fujisawa, N. (1992). On the torque mechanism of Savonius rotors. Journal of Wind Engineering and Industrial Aerodynamics, 40, pp. 277-292.

Gawade, S. G., \& Patil, D. S. (2015). Comparative Study of a Single Stage Savonius with a Combined Savonius-Three Bladed Darrieus. International Jounal For Technological Research In Engineering, 2(5), pp. 542-545.

Hayashi, T., Li, Y., \& Hara, Y. (2005). Wind tunnel tests on a different phase three-stage Savonius rotor. JSME International Journal, 48(1), 9-16. 
Jeon, K. S., Jeong, J. I., Pan, J.-K., \& Ryu, K.-W. (2014). Effects of end plates with various shapes and sizes on helical Savonius wind turbines. Renewable Energy, 1-10.

Kothe, L. B. (2016). Estudocomparativo experimental e numérico sobre o desempenho de turbinas Savonius helicoidal e de duplo estágio. Dissertação de Mestrado, Universidade Federal do Rio Grande do Sul, Porto Alegre, RS, Brasil.

Oliveira, C. P. (2014). Análise do desempenho de uma turbina savonius helicoidal com torção de $180^{\circ}$ empregando simulação numérica. Dissertação de Mestrado, Universidade Federal do Rio Grande do Sul, Porto Alegre, RS, Brasil.

Roy, S., \& Saha, U. K. (2013). Computational study to assess the influence of overlap ratio on static torque characteristics of a vertical axis wind turbine. Procedia Engineering, 694-702.

Sharma, K. K., Gupta, R., \& Biswas, A. (2014). Performance Measurement of a Two-Stage TwoBladed Savonius Rotor. International Journal of Renewable Energy Research, 4(1), pp. 115121.

Shepherd, W., \& Zhang, L. (2017). Electricity generation using wind power (2 ed.). Singapore: World Scientific.

Solidworks. (2016). Flow Simulation 2016 Technical Reference.

Wenehenubun, F., Saputra, A., \& Sutanto, H. (2015). An experimental study on the performance of Savonius wind turbines related with the number of blades. Energy Procedia, 297-304.

\section{COMO CITAR ESTE ARTIGO:}

BIANCHIN, L. S., BECK, D., SEIDEL, D. J. (2020). Influência da razão de sobreposição no torque estático da turbina Savonius. Holos. 36(6), 1-13.

\section{SOBRE OS AUTORES}

\section{S. BIANCHIN}

Especialista em Fontes Alternativas de Energia pela Universidade Pitágoras Unopar e cursando Formação Pedagógica em Matemática na mesma instituição. Graduado em Engenharia Mecânica pelo Instituto Federal de Educação, Ciência e Tecnologia Sul-Rio-Grandense, Câmpus Passo Fundo (2019). Sua principal pesquisa, tema do seu Trabalho de Conclusão de Curso, envolve a análise da influência de diferentes geometrias para o aumento do desempenho de partida da turbina eólica tipo Savonius utilizando Dinâmica dos Fluidos Computacional. E-mail: lucas.sbianchin@ hotmail.com

ORCID ID: https://orcid.org/0000-0002-5015-4302

\section{BECK}

Possui graduação em Engenharia Mecanica pela Universidade Federal do Rio Grande do Sul (2003), mestrado em Engenharia Mecânica pela Universidade Federal do Rio Grande do Sul (2005) e doutorado em Engenharia Mecânica pela Universidade Federal do Rio Grande do Sul (2009). Atualmente é professor concursado do Instituto Federal de Educação, Ciência e Tecnologia Sul-Rio-Grandense(IFSUL). Tem experiência na área de Engenharia Mecânica, com ênfase em Mecânica dos Fluídos e Termodinâmica,

HOLOS, Ano 36, v.6, e9891, 2020 
atuando principalmente nos seguintes temas: transformações conformes, turbulência, equação de helmholtz, equação de Navier-Stokes, simetrias de lie. Possui experiência docente na área metalmecânica, Resistência dos materiais, Fundição, Tratamento Térmico, Materiais de construção mecânica, Manutenção, Máquinas Térmicas.Bolsista Desenvolvimento Tecnológico e Inovação no Exterior (DEJ) 2015. E-mail: daniel.beck@ @assofundo.ifsul.edu.br

ORCID ID: https://orcid.org/0000-0002-8529-7792

\section{J. SEIDEL}

Possui graduação em Matemática Licenciatura Plena pela Universidade Federal de Santa Maria (2004), mestrado em Modelagem Matemática pela Universidade Regional do Noroeste do Estado do Rio Grande do Sul (2006) e doutorado em Ensino de Ciências e Matemática pela Universidade Luterana do Brasil (2013). Atualmente é professor do Instituto Federal Sul-Rio-Grandense. Tem experiência na área de Matemática, com ênfase em Educação Matemática, atuando principalmente nos seguintes temas: Educação Matemática, Educação a Distância Online, Formação de Professores, Tecnologias Digitais em processos educativos. E-mail: denilson.seidel@ passofundo.ifsul.edu.br

ORCID ID: https://orcid.org/0000-0003-2610-9189

Editor(a) Responsável: Francinaide de Lima Silva Nascimento

Pareceristas Ad Hoc: JERONIMO SILVA E IGOR ALMEIDA

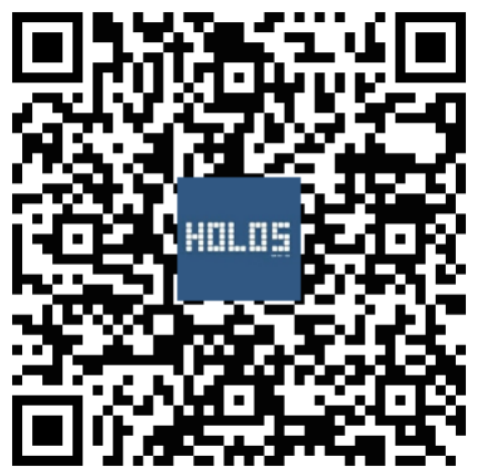

